5 Conway JB, Goldberg J, Chung F. Preadmission anaesthesia consultation clinic. Can J Anaesth 1992; 10: 1051-7.

6 Shevde $\mathrm{K}$, Panagopoulos G. A survey of 800 patients' knowledge, attitudes, and concerns regarding anesthesia. Anesth Analg 1991; 73: 190-8.

\section{Double lumen tube placement with the Pentax-Airway Scope®}

To the Editor:

Placement of double-lumen endobronchial tubes (DLT) in patients with difficult airways can present a major clinical challenge to the anesthesiologist. When glottic exposure is incomplete under direct laryngoscopy, tube placement may be achieved by blind probing for the glottic opening behind the epiglottis. However, a blind approach with a DLT can cause serious complications such as disclocation of the arytenoid cartilage, ${ }^{1}$ or airway trauma. Alternative techniques using the Bullard laryngoscope and other devices have been reported to facilitate DLT placement $^{2,3}$ but are not uniformly successful.

The Pentax-Airway Scope ${ }^{(}$(AWS) system is a novel intubation device which consists of a built-in monitor and camera, and an anatomically shaped blade holds a tube in the channel. The AWS improves the laryngeal view to facilitate tracheal intubation without requiring a laryngoscopist's line of sight. ${ }^{4}$

The AWS can be an excellent tool in the management of difficult intubations, and up to an $11-\mathrm{mm}$ external diameter tube can be set into its tube channel. The largest DLT available for the AWS is a size $32 \mathrm{Fr}$, which is not enough for adult patients requiring onelung ventilation. To address this limitation we remove the back plate of the tube channel for intubation with larger DLT (Figure).

We used this modified blade for a 39Fr DLT placement in a 56-yr-old male $(170 \mathrm{~cm}, 65 \mathrm{~kg})$. At the first intubation attempt, the laryngeal view obtained with Macintosh \#4 blade was a Cormack-Lehane grade 2b, and the bronchial lumen, but not the tracheal lumen, could be advanced into the trachea. Next, we bent the DLT to fit the contour of the AWS blade, held along with the AWS, which was inserted into the patient's oropharyngeal space. The DLT tip was continuously observed on the monitor to ensure avoidance of soft tissue injury during insertion. Visualization of the patient's vocal cords was achieved easily, the percentage of glottic opening score being $90 \%$, which is equivalent to Cormack Lehane grade 1 . Advancement to the bronchial lumen was achieved easily, followed

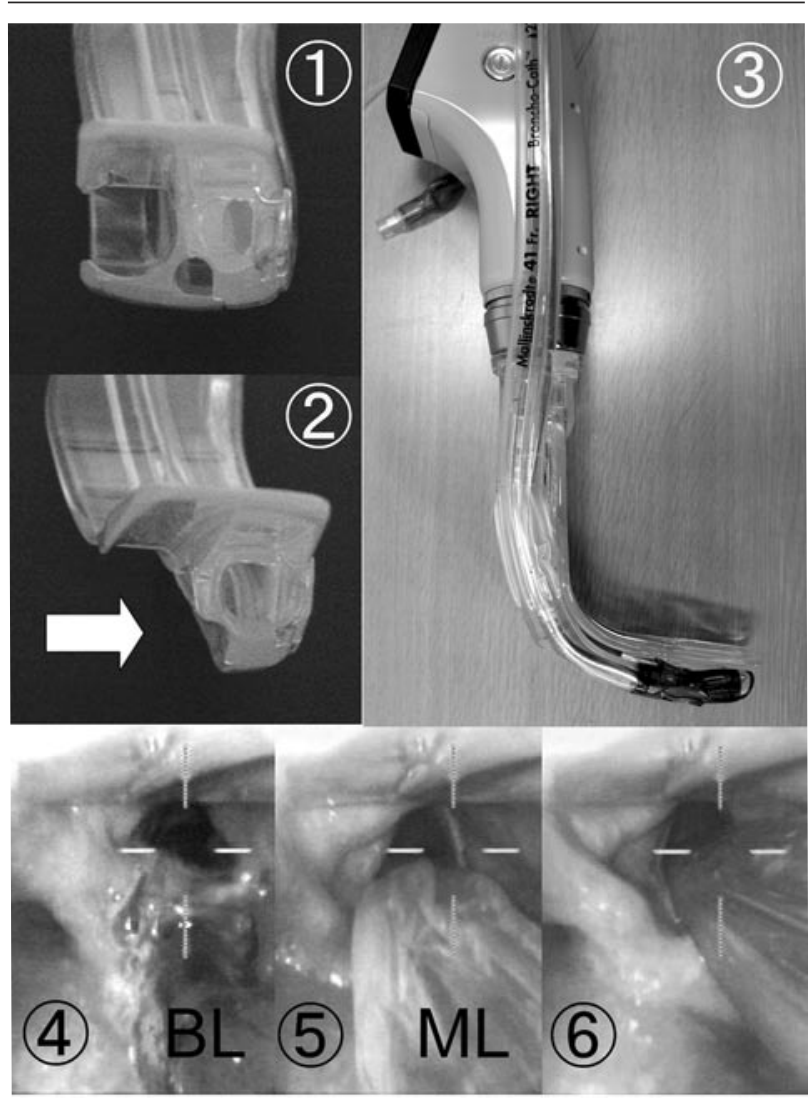

FIGURE 1) Photograph of the disposable blade (Pblade $\left.{ }^{\circledR}\right)$ tip for AWS. Up to 32 Fr DLT can be set in the tube channel. 2) Modified blade for DLT placement. Back plate of the tube channel was removed (white arrow). 3) AWS with 41 Fr DLT. A DLT stylet was bent to fit the contour of the Pblade ${ }^{\circledR}$. 4) Laryngeal view shown on the AWS's built-in monitor. Bronchial lumen (BL) is entering into the trachea. 5) The main lumen (ML) is entering into the trachea. 6) DLT placed within the trachea at the optimal depth. DLT = double-lumen endobronchial tube; AWS = Pentax-Airway Scope ${ }^{\circledR}$.

by tracheal lumen insertion with gentle counterclockwise rotation of the DLT. The DLT was placed successfully under indirect vision within $40 \mathrm{sec}$.

This is a similar approach to that reported with the Glidescope. ${ }^{5}$ However, the AWS may have several advantages over the Glidescope since the DLT can fit into the space behind the blade (originally it was a tube channel), Additionally, continuous observation of the tube tip can be achieved, and the laryngeal view is always improved to grade 1 by direct elevation of the epiglottis, as with Miller laryngoscope blades. ${ }^{3}$ 
Akihiro Suzuki MD PhD

Takayuki Kunisawa MD PhD

Hiroshi Iwasaki MD PhD

Asahikawa Medical College, Hokkaido, Japan

E-mail: masuikasuzuki@yahoo.co.jp

Accepted for publication July 9, 2007.

\section{References}

1 Mikuni I, Suzuki A, Takabata O, Fujita S, Otomo S, Iwasaki $H$. Arytenoid cartilage dislocation caused by a double-lumen endobronchial tube. Br J Anaesth 2006; 96. 136-8.

2 Shulman GB, Connelly NR. Double lumen tube placement with the Bullard laryngoscope. Can J Anesth 1999; 46: 232-4.

3 Hagibira S, Takashina M, Mori T, Yoshiya I. Onelung ventilation in patients with difficult airways. J Cardiothorac Vasc Anesth 1998; 12: 186-8.

4 Suzuki A, Toyama $\Upsilon$, Katsumi N, et al. Pentax-AWS ${ }^{\circledR}$ improves laryngeal view compared to Macintosh blade during laryngoscopy and facilitates easier intubation (Japanese). Masui 2007; 56: 464-8.

5 Hernandez AA, Wong DH. Using a Glidescope for intubation with a double lumen endotracheal tube. Can J Anesth 2005; 52: 658-9.

\section{Neck auscultation: a simple new method for confirming tracheal intubation}

To the Editor:

The current gold standards for confirmation of successful tracheal intubation include visualization of the endotracheal tube (ETT) passing through the vocal cords and end-tidal capnography. ${ }^{1}$ Mizutani et $a l^{2}$ could not distinguish between esophageal and tracheal intubation by auscultation at the suprasternal notch using spectral edge sound frequency analysis. An alternative approach is based on auscultation with a traditional stethoscope diaphragm at the level of the cricoid cartilage in the neck. Initial observations of this clinical sign were made with a Litmann ${ }^{\mathrm{TM}}$ Cardiology II SE stethoscope placed adjacent to the cricoid cartilage on the left side of the neck (Figure). The tip of an ETT (Mallincrodt Hi-Lo ${ }^{\circledR}$, Tyco Healthcare/ Mallinckrodt, Mansfield, CT, USA), upon striking the cricoid cartilage and tracheal rings, produces a classic sound akin to rubbing a pencil along standard plastic corrugated anesthesia tubing. The resulting sound is unique to the architecture and design of the cricoid cartilage and tracheal rings. For years we have utilized the experienced nurse in the operating room, to verify

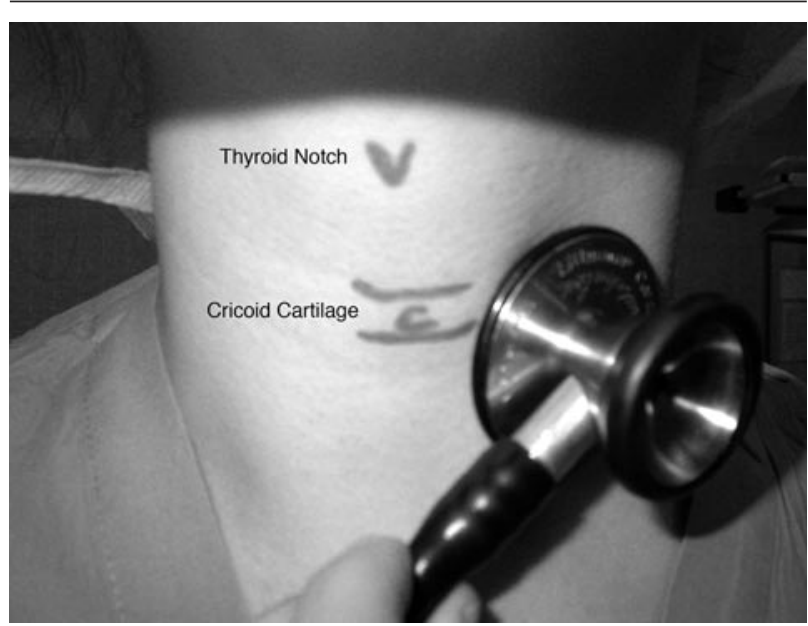

FIGURE Neck auscultation.

correct passage of an ETT into the trachea during the application of cricoid pressure. The tactile sensation generated when the ETT strikes the cricoid cartilage and subsequent tracheal rings is distinctly audible.

The stethoscope is placed adjacent to the cricoid cartilage on the left side of the neck as shown in the Figure. After tracheal intubation the lubricated ETT, with the balloon deflated, is gently advanced and withdrawn twice over a distance of $1-2 \mathrm{~cm}$, whilst auscultation is performed. This maneuver reproduces the previously described sounds when the trachea has been intubated. Posterior displacement of the ETT in the direction of the hard palate augments the anterior position of the tube tip within the trachea, and hence the sounds generated. Application of cricoid pressure may further augment the sounds originating from the ETT tip striking the anterior cricoid and tracheal cartilages.

This auscultatory method of confirming tracheal tube placement can serve as an additional clinical guide to exclude esophageal intubation. The maneuver may be potentially beneficial in emergency resuscitation outside of the controlled operating room setting, and should be considered whenever doubt exists regarding correct ETT placement.

Chris Christodoulou MBChB FRCPC

University of Manitoba, Winnipeg, Canada

E-mail: chrischristodoulou@shaw.ca

Accepted for publication June 17, 2007.

\section{References}

1 American Society of Anesthesiologists Task Force on 